\title{
Two-dimensional particle-in-cell Monte Carlo simulation of a miniature inductively coupled plasma source
}

\section{$\operatorname{AUTHOR}(\mathrm{S}):$}

Takao, Yoshinori; Kusaba, Naoki; Eriguchi, Koji; Ono, Kouichi

\section{CITATION:}

Takao, Yoshinori ... [et al]. Two-dimensional particle-in-cell Monte Carlo simulation of a miniature inductively coupled plasma source. Journal of Applied Physics 2010, 108(9): 093309.

\section{ISSUE DATE:}

2010-11

URL:

http://hdl.handle.net/2433/134553

RIGHT:

(C) 2010 American Institute of Physics 


\title{
Two-dimensional particle-in-cell Monte Carlo simulation of a miniature inductively coupled plasma source
}

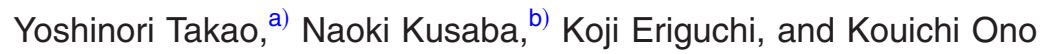 \\ Department of Aeronautics and Astronautics, Graduate School of Engineering, Kyoto University, \\ Yoshida-Honmachi, Sakyo-ku, Kyoto 606-8501, Japan
}

(Received 27 August 2010; accepted 29 September 2010; published online 10 November 2010)

\begin{abstract}
Two-dimensional axisymmetric particle-in-cell simulations with Monte Carlo collision calculations (PIC-MCC) have been conducted to investigate argon microplasma characteristics of a miniature inductively coupled plasma source with a 5-mm-diameter planar coil, where the radius and length are $5 \mathrm{~mm}$ and $6 \mathrm{~mm}$, respectively. Coupling the rf-electromagnetic fields to the plasma is carried out based on a collisional model and a kinetic model. The former employs the cold-electron approximation and the latter incorporates warm-electron effects. The numerical analysis has been performed for pressures in the range $370-770$ mTorr and at $450 \mathrm{MHz}$ rf powers below $3.5 \mathrm{~W}$, and then the PIC-MCC results are compared with available experimental data and fluid simulation results. The results show that a considerably thick sheath structure can be seen compared with the plasma reactor size and the electron energy distribution is non-Maxwellian over the entire plasma region. As a result, the distribution of the electron temperature is quite different from that obtained in the fluid model. The electron temperature as a function of rf power is in a reasonable agreement with experimental data. The pressure dependence of the plasma density shows different tendency between the collisional and kinetic model, implying noncollisional effects even at high pressures due to the high rf frequency, where the electron collision frequency is less than the rf driving frequency. (C) 2010 American Institute of Physics. [doi:10.1063/1.3506536]
\end{abstract}

\section{INTRODUCTION}

In recent years, microplasma sources have attracted increasing attention, aimed at many applications, such as micropropulsion, ${ }^{1-7}$ plasma displays ${ }^{8,9}$ miniature mass spectrometers, ${ }^{10}$ biomedical applications, ${ }^{11}$ and so on. Such microplasma sources are obtained by dc plasma, capacitively coupled plasma, inductively coupled plasma, and microwave-excited plasma. In order to broaden the application fields, it is crucial to understand microplasma characteristics profoundly. For measurements of plasma parameters in a small space less than a few millimeters, the Langmuir probe method is very challenging so that optical diagnostics are widely used. ${ }^{9}$ However, the spatial distribution of plasma parameters is not easily available in experiments. On the other hand, numerical simulations can also be useful, and many fluid and particle simulations were already performed. ${ }^{2,6,7,12-15}$

Fluid simulations are easy to handle in the system involving many reactions and require rather short computational time compared with particle simulations. Although most fluid models are developed assuming Maxwellian distribution, the electron energy distribution is usually far from equilibrium in microplasmas. ${ }^{15}$ Since most reactions are caused by high energy electrons, information of the electron energy distribution is quite important, which can be treated with particle models. There are few papers on two- and three-dimensional particle models compared with one-

\footnotetext{
${ }^{a)}$ Electronic mail: ystakao@aero.mbox.media.kyoto-u.ac.jp.

${ }^{b}$ Present address: Mitsubishi Heavy Industries, Ltd., Nagoya Aerospace Systems Works, Oye-cho, Minato-ku, Nagoya 455-8515, Japan.
}

dimensional ones. In order to obtain spatial distributions of plasma parameters and electron kinetic information in detail, further numerical study is required based on particle models with more than one dimension.

In the present work, we have developed a twodimensional axisymmetric particle model employing particle-in-cell calculations with a Monte Carlo collision method (PIC-MCC) for a miniature inductively coupled plasma (mICP) source. The mICP generator with a $5-\mathrm{mm}-$ diameter planar coil was fabricated and investigated experimentally by Hopwood et al., ${ }^{16}$ and Nam and Economou ${ }^{14}$ presented a two-dimensional fluid simulation for the mICP in Ar assuming Maxwellian electrons. We describe the particle model developed in Sec. II. Results and discussion are then presented in Sec. III, where we compare our results with experimental data ${ }^{16}$ and fluid simulation results, ${ }^{14}$ indicating that the electron energy distribution is non-Maxwellian. Finally, conclusions are drawn in Sec. IV.

\section{NUMERICAL MODEL}

Figure 1 shows a schematic of the mICP source with the 5 -mm-diameter planar coil. ${ }^{16}$ The microplasma source is composed of a cylindrical dielectric tube, the inner radius and the length of which are $5 \mathrm{~mm}$ and $6 \mathrm{~mm}$, respectively. The three-turn coil is located on the top of a 700- $\mu$ m-thick dielectric and the bottom of the plasma source is bounded with a metal wall. The Ar plasma is generated by the rf current applied to the coil at a high frequency of $450 \mathrm{MHz}$ for relatively high pressures of 100s mTorr, compared with conventional ICP sources. The simulation area is only Ar plasma region. The area is divided into 60 cells in the axial 


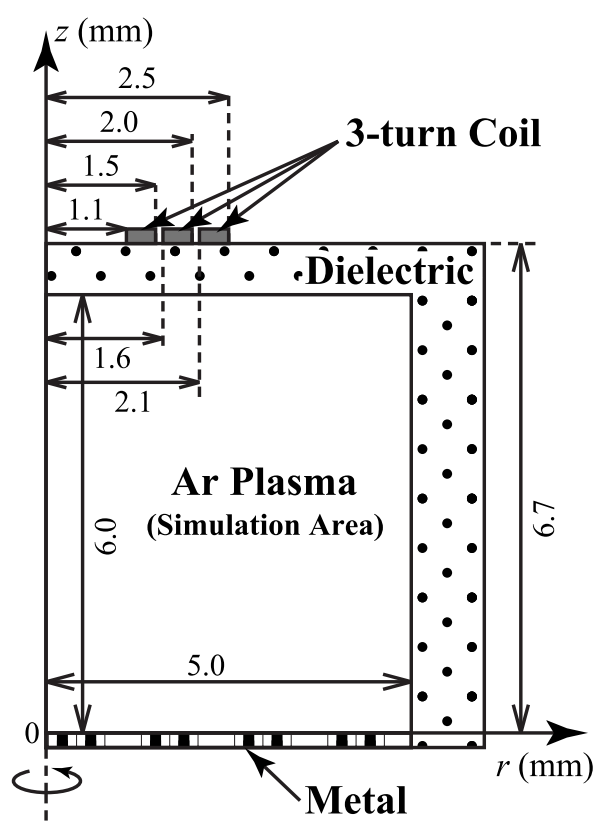

FIG. 1. Schematic of the mICP source with the 5-mm-diameter planar coil. Simulation area is only Ar plasma region; $6 \mathrm{~mm}$ in length, and $5 \mathrm{~mm}$ in radius. The area is divided into 60 cells in the axial direction and 50 cells in the radial direction at regular intervals. The cylindrical coordinates $(r-z)$ are employed, with the origin being placed on the $z$ axis at the bottom of the plasma source.

direction and 50 cells in the radial direction at regular intervals. The cylindrical coordinates $(r-z)$ are employed, with the origin being placed on the $z$ axis at the bottom of the plasma source. The present model consists of the electromagnetic equation for the rf-induced azimuthal electric field, Poisson's equation for the electrostatic field owing to the space charge, and the equation of motion and collisions of charged particles. ${ }^{17}$

\section{A. Assumptions}

To investigate the characteristics of the mICP source, we have conducted PIC-MCC, ${ }^{18,19}$ assuming the following conditions.

(i) Only $\mathrm{Ar}$ ions and electrons are treated as particles, and the ion species of interest is singly-ionized $\mathrm{Ar}^{+}$ only.

(ii) Neutral particles are spatially uniform throughout the simulation with a Maxwellian velocity distribution at a gas temperature of $300 \mathrm{~K}(=0.026 \mathrm{eV})$.

(iii) The reactions taken into account are elastic, excitation, and ionization collisions for electrons, and elastic and charge exchange collisions for ions, as below. $^{20}$

$$
\begin{aligned}
& \mathrm{e}+\mathrm{Ar} \rightarrow \mathrm{e}+\mathrm{Ar} \quad(\text { Elastic scattering) } \\
& \mathrm{e}+\mathrm{Ar} \rightarrow \mathrm{e}+\mathrm{Ar}^{*} \quad(\text { Excitation }), \\
& \mathrm{e}+\mathrm{Ar} \rightarrow \mathrm{e}+\mathrm{Ar}^{+}+\mathrm{e} \quad(\text { Ionization) } \\
& \mathrm{Ar}^{+}+\mathrm{Ar} \rightarrow \mathrm{Ar}^{+}+\mathrm{Ar} \quad \text { (Elastic scattering), }
\end{aligned}
$$
$\mathrm{Ar}^{+}+\mathrm{Ar} \rightarrow \mathrm{Ar}+\mathrm{Ar}^{+}$
(Charge exchange).

(iv) The motion of excited-state atoms is not considered.

(v) Coulomb collisions are not taken into account.

(vi) The coordinate system is axisymmetric, in which a number of simulated particles (or superparticles for ions and electrons) are loaded in a two-dimensional spatial mesh $(r, z)$, along with three velocity components $\left(v_{r}, v_{\theta}, v_{z}\right)$.

(vii) Capacitive coupling from the rf antenna is not treated.

\section{B. Electromagnetic Field}

The mICP is generated by the rf current applied to the antenna coil. All wave quantities, such as the electromagnetic field and current density, are assumed to vary harmonically in time as $e^{i \omega t}$, where $i$ is the square root of $-1, t$ is the time, and $\omega$ is the rf angular frequency. We also assume that the coil is composed of three concentric rings, so that the electric field has only the azimuthal component. Then, the induced electric field $E_{\theta}$ and the plasma current density $j_{\theta}$ are set to be $E_{\theta}=\widetilde{E}_{\theta} e^{i \omega t}$ and $j_{\theta}=\widetilde{J}_{\theta} e^{i \omega t}$, respectively, where $\widetilde{E}_{\theta}$ and $\widetilde{J}_{\theta}$ are the complex amplitude. The complex amplitude of the electromagnetic fields is obtained from the following equation. ${ }^{17,21-23}$

$$
\left(\frac{\partial^{2}}{\partial r^{2}}+\frac{1}{r} \frac{\partial}{\partial r}+\varepsilon_{0} \mu_{0} \omega^{2}-\frac{1}{r^{2}}+\frac{\partial^{2}}{\partial z^{2}}\right) \widetilde{E}_{\theta}=i \omega \mu_{0} \widetilde{J}_{\theta}
$$

where $\varepsilon_{0}$ is the electric permittivity of vacuum and $\mu_{0}$ is the magnetic permeability of vacuum. The boundary conditions of $\widetilde{E}_{\theta}$ are set to be zero at the metal wall assuming perfectly conducting materials and on the $z$ axis $(r=0)$ due to the axisymmetry. On the plasma-dielectric window interfaces the electric field is analytically derived from Biot-Savart's law, which is the sum of the fields over the three-turn coil current and the plasma current. ${ }^{21-24}$ The magnetic field $\mathbf{B}$ is then obtained from Faraday's law with the electric field determined by Eq. (1).

\section{Electrostatic field}

The potential $\phi$ and electrostatic field $\mathbf{E}$ due to the space charge are given by

$$
\begin{aligned}
& \left(\frac{\partial^{2}}{\partial r^{2}}+\frac{1}{r} \frac{\partial}{\partial r}+\frac{\partial^{2}}{\partial z^{2}}\right) \phi=-\frac{\rho(r, z)}{\varepsilon_{0}}, \\
& \mathbf{E}=-\nabla \phi,
\end{aligned}
$$

where $\rho$ is the charge density. Using the superposition principle, we divide the potential $\phi$ into two types of potentials, i.e., $\phi=\phi_{L}+\phi_{P}$, where $\phi_{L}$ is the solution of the Laplace equation and $\phi_{P}$ is that of the Poisson equation. ${ }^{17,25}$ The potential $\phi_{P}$ is derived from the space charge of charged particles and can be obtained from Eq. (2) with zero potential boundary conditions except on the $z$ axis $(r=0)$, where zero radial potential gradient condition is imposed: $\partial \phi_{P} / \partial r=0$ due to the axisymmetry. The potential $\phi_{L}$ is obtained from Eq. (2) with the right-hand side of it being set at zero. The boundary conditions of $\phi_{L}$ are zero potential on the metal 
wall and $\partial \phi_{L} / \partial r=0$ at $r=0$. On the dielectric, the potential $\phi_{L}$ satisfies the following equations: ${ }^{26,27}$

$$
\begin{aligned}
& E_{d}=\frac{\sigma_{d}}{\varepsilon_{0}}, \\
& E_{d}=-\frac{\partial \phi_{L}}{\partial n},
\end{aligned}
$$

where $\partial / \partial n$ is the derivative normal to the dielectric and the subscript $d$ represents the values thereat. The surface charge $\sigma_{d}$ on the dielectric is obtained from the summation of the charged particles incident thereon. The electrostatic field $\mathbf{E}$ is then determined as $\mathbf{E}=-\nabla\left(\phi_{L}+\phi_{P}\right)$ from Eq. (3). Here, to eliminate a systematic error in charge density on the $z$ axis $(r=0)$ for cylindrical coordinates, we employ a general weighting scheme presented by Verboncoeur. ${ }^{28}$ We also apply a digital smoothing algorithm to the space charge in order to decrease the numerical noise owing to the limited number of superparticles. ${ }^{19,29}$

\section{Motion and collisions of charged particles}

Using the electromagnetic and electrostatic fields obtained above, we move charged particles by integrating the equation of motion.

$$
\begin{aligned}
& \frac{d \mathbf{v}}{d t}=\frac{q}{m}(\mathbf{E}+\mathbf{v} \times \mathbf{B}), \\
& \frac{d \mathbf{x}}{d t}=\mathbf{v},
\end{aligned}
$$

where $\mathbf{v}$ is the velocity, $q$ is the charge, $m$ is the mass of a particle, and $\mathbf{x}$ is the position. Equations (6) and (7) are solved explicitly by a time-centered leap frog method for time integration and Buneman-Boris method for the $\mathbf{v} \times \mathbf{B}$ rotation term. ${ }^{19}$ The collision of charged particles can be treated separately from the calculation of motion as long as a chosen time step is much smaller than the mean free time. ${ }^{30}$ To reduce the calculation time, we employ the null-collision method $^{20}$ in MCC with cross sections for electrons ${ }^{31,32}$ and ions. ${ }^{20,33}$ The postcollision velocities of charged particles are determined by the conservation equations of momentum and energy. ${ }^{30}$ In order to speed up the simulation, ions motion and collisions are calculated only once per ten electrons time steps owing to their difference in the speed of motion. Here, the numerical time step $\Delta t$ is taken to be $1.1 \times 10^{-12} \mathrm{~s}(1 /$ 2000 of an rf cycle for $450 \mathrm{MHz}$ ) for electrons and 1.1 $\times 10^{-11} \mathrm{~s}$ for ions, respectively.

\section{E. Power deposition in the plasma}

To solve Eq. (1), the relation between $\widetilde{J}_{\theta}$ and $\widetilde{E}_{\theta}$ should be specified. The cold-electron approximation is commonly used, i.e., the current density is given by Ohm's law.

$$
\widetilde{J}_{\theta}=\sigma_{p} \widetilde{E}_{\theta} .
$$

Here, $\sigma_{p}$ is the plasma conductivity and defined as follows: ${ }^{34}$

$$
\sigma_{p}=\frac{\varepsilon_{0} \omega_{p e}^{2}}{i \omega+\nu_{m}}=\frac{q^{2} n_{e}}{m_{e}}\left(\frac{\nu_{m}}{\nu_{m}^{2}+\omega^{2}}-i \frac{\omega}{\nu_{m}^{2}+\omega^{2}}\right)
$$

where $\omega_{p e}, \nu_{m}, n_{e}$, and $m_{e}$ denote the electron plasma frequency, electron momentum transfer collision frequency, electron density, and electron mass, respectively. In our calculations, the collision frequency $\nu_{m}$ is determined by the following equation. ${ }^{35}$

$$
\nu_{m}=\frac{n_{g} \int \mathbf{v} \sigma_{m} f(\mathbf{v}) d \mathbf{v}}{\int f(\mathbf{v}) d \mathbf{v}},
$$

where $n_{g}$ is the background Ar gas density, $\sigma_{m}$ is the cross section of momentum transfer collisions for electrons, ${ }^{32}$ and $f(\mathbf{v})$ is the velocity distribution functions of electrons. The ion current can be ignored owing to the low mobility and then we can solve Eq. (1) using Eqs. (8)-(10). The timeaveraged collisional power deposition per unit volume $P_{c}$ is given by

$$
P_{c}=\frac{1}{2} \operatorname{Re}\left(\sigma_{p}\right)\left|\widetilde{E}_{\theta}\right|^{2},
$$

where $\operatorname{Re}\left(\sigma_{p}\right)$ is the real part of the plasma conductivity. Equation (11) represents that the only ohmic heating is considered as the mechanism of electron heating. Here, we refer to the analysis based on the cold-electron approximation as the collisional model.

In the particle model, one can derive the plasma current density directly by following electron trajectories. This calculation is fully kinetic and no assumptions are required about the mechanism of electron heating. The kinetic plasma current density is obtained from the equation below. ${ }^{17,36}$

$$
\widetilde{J}_{\theta}=\frac{1}{V_{g}} \Sigma\left(-q W_{e} v_{\theta}\right) \exp (i \Delta \psi),
$$

where $V_{g}$ is the cell volume centered at a grid point, $W_{e}$ is the weight of a electron superparticle (i.e., the number of physical electrons per computational particle for electrons), $v_{\theta}$ is the amplitude of the azimuthal component of the electron velocity at the fundamental frequency, $\Sigma$ is the summation of all the electron superparticles in the volume $V_{g}$, and $\Delta \psi$ is the phase difference between the rf coil current $I_{\text {coil }}$ and $j_{\theta}$. The phase difference $\Delta \psi$ is expressed as $\Delta \psi_{1}+\Delta \psi_{2}: \Delta \psi_{1}$ is the phase difference between $I_{\text {coil }}$ and $E_{\theta}$, which is obtained from the ratio of the real part of $E_{\theta}$ to the imaginary part of $E_{\theta}$, and $\Delta \psi_{2}$ is the phase difference between $E_{\theta}$ and $j_{\theta}$. To determine $\Delta \psi_{2}$, we carry out a discrete fast Fourier transform for $j_{\theta}$ to isolate the component at the fundamental harmonic and then calculate its inverse transform. Once the phase difference $\Delta \psi$ is obtained, the electric field $E_{\theta}$ can be determined with Eqs. (1) and (12). The time-averaged kinetic power deposition per unit volume $P_{k}$ is calculated from

$$
P_{k}=\frac{1}{\tau} \int_{\tau} \frac{1}{V_{g}} \Sigma\left(-q W_{e} \mathbf{v}_{e} \cdot \mathbf{E}\right) d t,
$$

where $\tau$ is the multiple of a rf period and $\mathbf{v}_{e}$ is the electron velocity. ${ }^{36}$ This implementation enables us to selfconsistently take into consideration the effect of noncollisional heating, which is important to low pressure rf plasmas. 
Here, we refer to the analysis based on Eqs. (12) and (13) as the kinetic model.

\section{F. Additional remarks}

Initially, the electron and ion superparticles with Maxwellian velocities are uniformly distributed in the simulation area. ${ }^{25}$ The initial numbers of electron and ion superparticles are set at 950000 , respectively. The same initial conditions are employed in all the calculations below. The superparticles increase through the ionization process and decrease owing to the incident on the walls through the calculation. The number of ion superparticles is counted at each rf cycle, and, if the number exceeds or falls below a predefined limit, the number of superparticles is adjusted to about the initial number (between 900000 and 100000 0). Correspondingly, the weight of the remaining superparticles is decreased or increased to conserve the number density of charged particles. ${ }^{25}$ Here, the weight is constant spatially. Since we focus on the results at steady state, the rf electromagnetic fields are updated every ten rf cycles to reduce the calculation cost, and also to minimize the numerical noise of the plasma current density in Eq. (12) for the kinetic model. In the simulation, the total power deposition is used as an input parameter, which is obtained by integrating Eq. (11) or Eq. (13) over the entire simulation area. Thus, we rescale $I_{\text {coil }}$ and $\widetilde{E}_{\theta}$ to yield the specified total power deposition until the steady state solution is obtained. The macroscopic parameters, such as the electron density and electron temperature, shown in Sec. III, were determined by averaging over 1000 rf cycles after the steady state was reached.

\section{RESULTS AND DISCUSSION}

The particle simulations for both the collisional and the kinetic model were conducted at an $\mathrm{Ar}$ pressure $p$ $=500 \mathrm{mTorr}$, rf frequency $f=450 \mathrm{MHz}$, rf power $P_{\mathrm{rf}}$ $=1.3 \mathrm{~W}$, and power transfer efficiency $\eta=2.5 \%$, where the total power deposition in the plasma is calculated to be 32.5 $\mathrm{mW}(2.5 \%$ of $1.3 \mathrm{~W})$. The simulation condition above is same as the base case condition in the fluid model. ${ }^{14}$ In such mICP sources, the power transfer efficiency was determined to be less than a few percents below 1 Torr because most of the rf power was dissipated in the microfabricated circuit. ${ }^{37}$ Note that the volume-averaged power density at $32.5 \mathrm{~mW}$ for the mICP source shown in Fig. 1 is calculated to be 6.9 $\times 10^{-2} \mathrm{~W} / \mathrm{cm}^{3}$, which is comparable to that for conventional ICP sources. ${ }^{17}$

Figure 2 shows the two-dimensional distribution of the absorbed power density in the mICP source. Most of the rf power is deposited locally near the coil in a toroidal pattern. The peak power density in the kinetic model is $1.1 \mathrm{~W} / \mathrm{cm}^{3}$, which is somewhat larger than that in the collisional model, and its position is slightly closer to the coil with a steeper gradient. The distribution in the kinetic model is similar to that in the collisional model except near boundaries, where negative power deposition can be seen because of the electron diffusion toward the walls. The increase in power deposition near the $z$ axis is probably due to the statistical fluctuation since we use a spatially uniform weight of

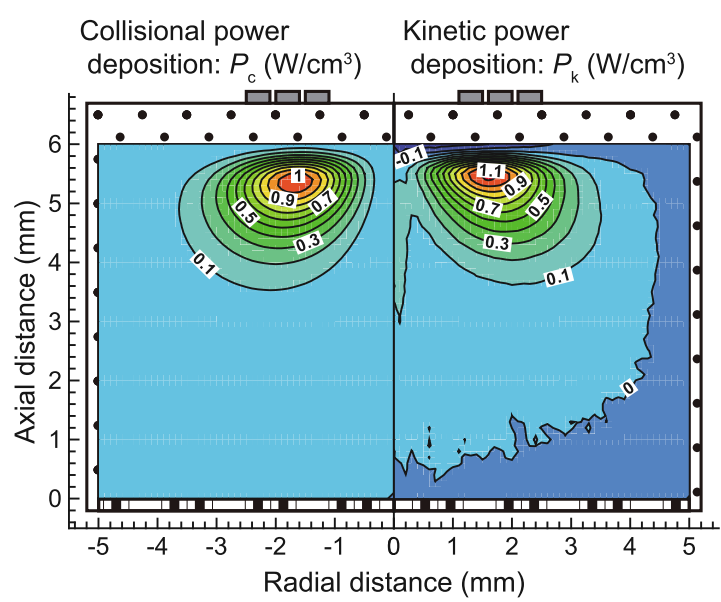

FIG. 2. (Color online) Two-dimensional distribution of the time-averaged absorbed power density in the mICP source based on the collisional model (left) and the kinetic model (right), calculated at an $\mathrm{Ar}$ pressure $p$ $=500$ mTorr, rf frequency $f=450 \mathrm{MHz}$, rf power $P_{\mathrm{rf}}=1.3 \mathrm{~W}$, and power transfer efficiency $\eta=2.5 \%$.

superparticles for the cylindrical coordinates. However, the influence of the fluctuation on the total power deposition is negligible owing to the small volume near $r=0$. The fluctuation would be avoided using a weighting factor presented by Takekida and Nanbu. ${ }^{38}$

Figure 3 shows the two-dimensional distribution of the effective electron temperature in the mICP source. Here, the effective electron temperature $T_{\text {eff }}$ was calculated as below.

$$
T_{\text {eff }}=\frac{2}{3} \int \varepsilon f_{d}(\varepsilon) d \varepsilon
$$

where $\varepsilon$ is the electron energy in electron volt and $f_{d}(\varepsilon)$ is a normalized electron energy distribution function, i.e., $\int f_{d}(\varepsilon) d \varepsilon=1 .{ }^{39}$ The distributions are almost the same for both the models. The peak $T_{\text {eff }}$ is obtained at the position close to where the peak power deposition is obtained in Fig. 2. While the absorbed power is localized near the coil, $T_{\text {eff }}$ keeps high values in the bulk plasma region. Since electrons heated near the coil diffuse to the bulk region and lose their energy

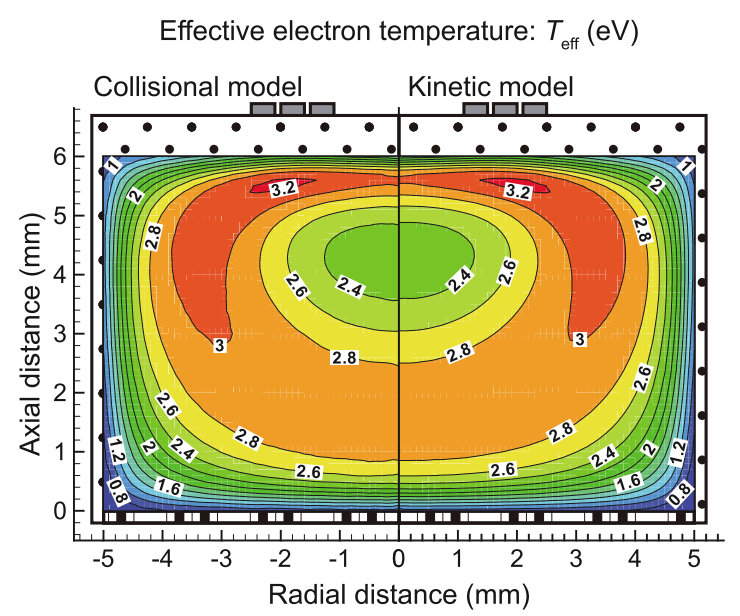

FIG. 3. (Color online) Two-dimensional distribution of the time-averaged effective electron temperature $T_{\text {eff }}$ in the mICP source based on the collisional model (left) and the kinetic model (right) under the same conditions as those in Fig. 2. 
Electron density: $n_{\mathrm{e}}\left(10^{10} \mathrm{~cm}^{-3}\right)$

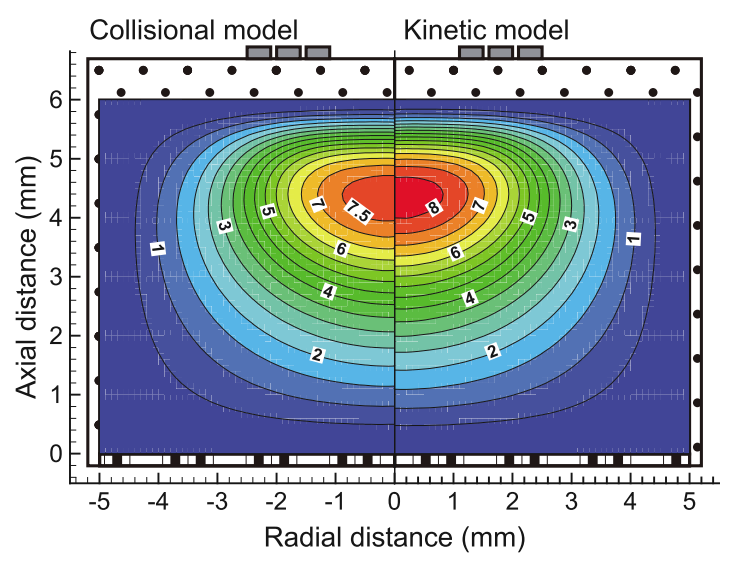

FIG. 4. (Color online) Two-dimensional distribution of the time-averaged electron density $n_{e}$ in the mICP source based on the collisional model (left) and the kinetic model (right) under the same conditions as those in Fig. 2.

through collisions, $T_{\text {eff }}$ decreases around the center of the mICP source, where the flat potential is obtained (see Fig. 5). The effect of the ambipolar diffusion increases $T_{\text {eff }}$ near the plasma-sheath interface and deceleration of electrons decreases $T_{\text {eff }}$ near the walls due to the potential barrier. Notice that the distribution of $T_{\text {eff }}$ is quite different from that obtained in the fluid model (see Fig. 3 in Ref. 14).

Figure 4 shows the two-dimensional distribution of the electron density $n_{e}$ in the mICP source. The peak $n_{e}$ in the kinetic model is $8.2 \times 10^{10} \mathrm{~cm}^{-3}$, which is slightly larger than that in the collisional model $\left(7.8 \times 10^{10} \mathrm{~cm}^{-3}\right)$, and its position is close to where the local minimum $T_{\text {eff }}$ is obtained in Fig. 3. The peak electron density is located on the $z$ axis close to the coil, not directly under the coil where the peak power deposition is obtained, owing to the diffusion effect and large surface-to-volume ratio of the mICP source. The steep gradients of $n_{e}$ clearly show that experimental investigations of spatial profiles cannot readily be done. Whereas the distribution of $T_{\text {eff }}$ is quite different from that obtained in the fluid model, the distribution of $n_{e}$ is similar to that in the fluid model (see Fig. 4 in Ref. 14).

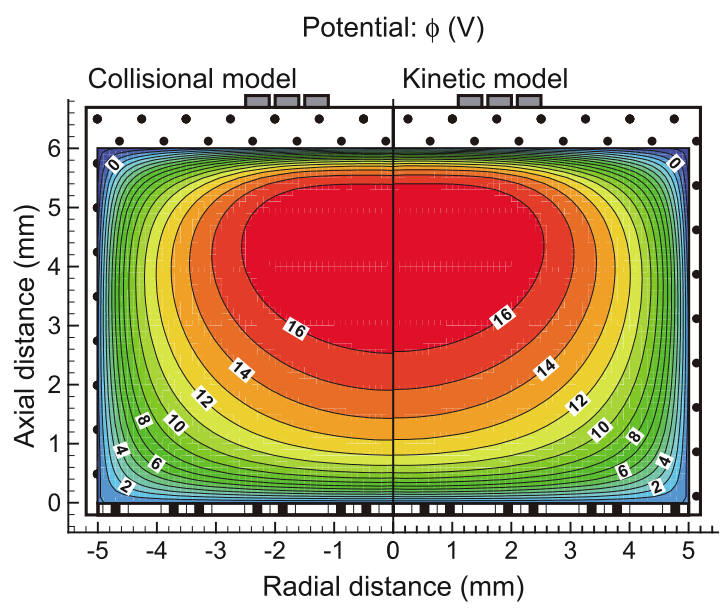

FIG. 5. (Color online) Two-dimensional distribution of the time-averaged potential $\phi$ in the mICP source based on the collisional model (left) and the kinetic model (right) under the same conditions as those in Fig. 2.

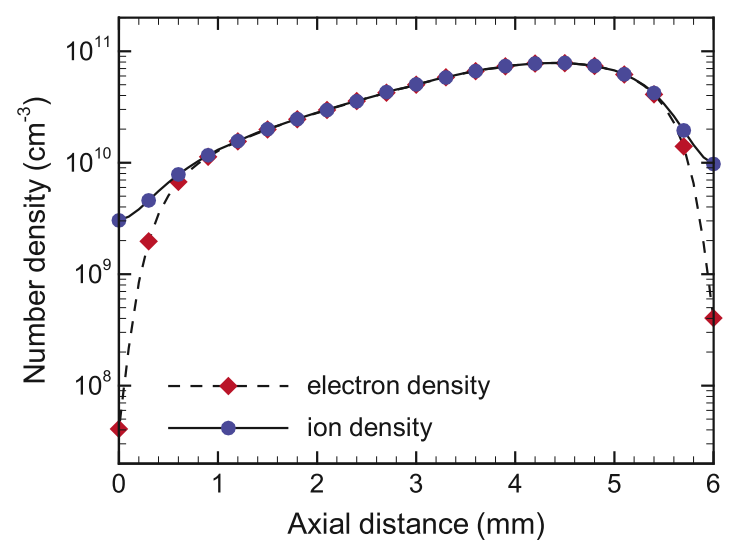

FIG. 6. (Color online) Axial distribution of the time-averaged electron density $n_{e}$ and ion density $n_{i}$ at $r=1 \mathrm{~mm}$ in the mICP source based on the kinetic model under the same conditions as those in Fig. 2. Notice that the three-turn coil is located at the axial distance $z=6.7 \mathrm{~mm}$ as shown in Fig. 1 .

Figure 5 shows the two-dimensional distribution of the potential $\phi$ in the mICP source. The distributions are also almost the same for both the models. The peak potential is obtained on the $z$ axis close to the coil, where the electron density exhibits peak values. While a relatively flat potential profile is shown in the bulk region, steep potential gradients are obtained near the boundaries. As stated in the assumptions, capacitive coupling from the rf antenna is not taken into account in the particle model developed, so that slightly negative potential is obtained on the dielectric boundaries. ${ }^{17}$ In conventional low pressure ICP sources large negative potential was observed at the dielectric window due to the large capacitive power coupling, resulting in the window damage. $^{40}$ Implementation of the capacitive coupling into our model is left for future work.

Figure 6 shows the axial distribution of the electron density $n_{e}$ and ion density $n_{i}$ at $r=1 \mathrm{~mm}$ in the mICP source under the same conditions as those in Fig. 2. Note that the three-turn coil is located at the axial distance $z=6.7 \mathrm{~mm}$. As clearly seen in Fig. 6, we can find a relatively thick sheath structure compared with the plasma reactor length. The sheath thickness can reach over $0.5 \mathrm{~mm}$, which is about $10 \%$ of the plasma reactor length, so that the sheath should not be neglected in the simulation for such mICP sources. ${ }^{37}$ Shown in Fig. 7 is the normalized electron energy probability function (EEPF) at various positions in the mICP source: near the coil boundary $(r=1.7, z=5.5 \mathrm{~mm})$, in the bulk plasma $(r$ $=1.7, z=3.0 \mathrm{~mm})$, and near the metal boundary $(r=1.7, z$ $=0.5 \mathrm{~mm}$ ). As shown in the figure, all EEPFs are nonMaxwellian. At higher electron energies, depletions of EEPFs are clearly seen due to the inelastic collisions. The depletions occur between excitation $\left(E_{\mathrm{ex}}=11.6 \mathrm{eV}\right)$ and ionization $\left(E_{\mathrm{iz}}=15.8 \mathrm{eV}\right)$ threshold energies, except the EEPF near the metal boundary, which is close to a Druyvesteyn distribution. Since the sheath structure was neglected and Maxwellian electrons were assumed in the fluid model, ${ }^{14}$ the fluid model could not express the electron temperature distribution correctly.

Since the Langmuir probe data were obtained at $r=0, z$ $=5 \mathrm{~mm}$ in the experiment, ${ }^{16}$ and the comparison of the fluid simulation with the experimental data was conducted at this 


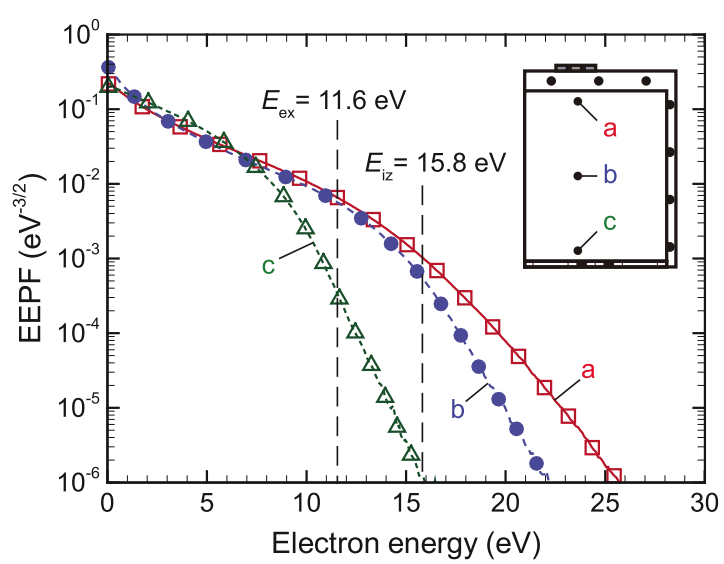

FIG. 7. (Color online) Normalized EEPF at various positions in the mICP source based on the kinetic model under the same conditions as those in Fig. 2. The positions are depicted in the inset: (a) $r=1.7, z=5.5 \mathrm{~mm}$; (b) $r$ $=1.7, z=3.0 \mathrm{~mm}$; and (c) $r=1.7, z=0.5 \mathrm{~mm}$. Here, $E_{\text {ex }}$ and $E_{\text {iz }}$ are the excitation threshold energy and the ionization threshold energy, respectively.

point, ${ }^{14}$ we also compared our calculation results with the experiment and fluid simulation results at the same point. Figure 8 shows the comparison among the experimental data, fluid simulation, and particle simulation of the electron temperature as a function of rf power for $p=500 \mathrm{mTorr}$. Note that we performed calculations at a fixed power transfer efficiency $\eta=2.5 \%$ for all the rf powers. The electron temperature is almost constant for both the fluid model and the particle model. The values are lower than the experimental result by $0.4-1.0 \mathrm{eV}$ whereas the experimental data are also independent of $\mathrm{rf}$ power. In view of the fact that accurate measurements of the electron temperature with Langmuir probe are difficult, particularly for microplasmas, ${ }^{9}$ the simulation results are in good agreement with the experiment.

Figure 9 shows the comparison among the experimental data, fluid simulation, and particle simulation of the electron density as a function of rf power for $p=500 \mathrm{mTorr}$ and $\eta$ $=2.5 \%$. The electron density in the particle model increases linearly with increasing rf power, resulting in a large gradient compared with the experiment and fluid model results. However, the power transfer efficiency decreased from $5 \%$ to $2 \%$ with increasing rf power from 0.75 to $2.5 \mathrm{~W}$ at $700 \mathrm{mTorr}$ in

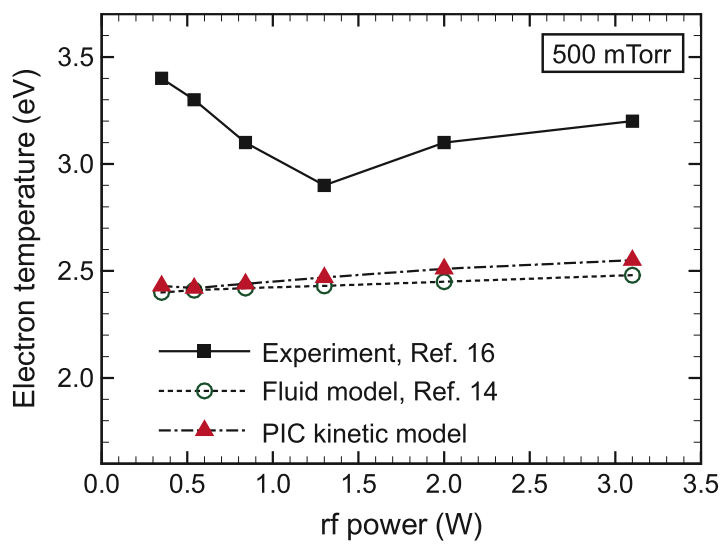

FIG. 8. (Color online) Electron temperature as a function of $\mathrm{rf}$ power at an Ar pressure $p=500 \mathrm{mTorr}$ and power transfer efficiency $\eta=2.5 \%$ for comparison among the experiment (squares), fluid simulation (circles), and particle simulation based on the kinetic model (deltas).

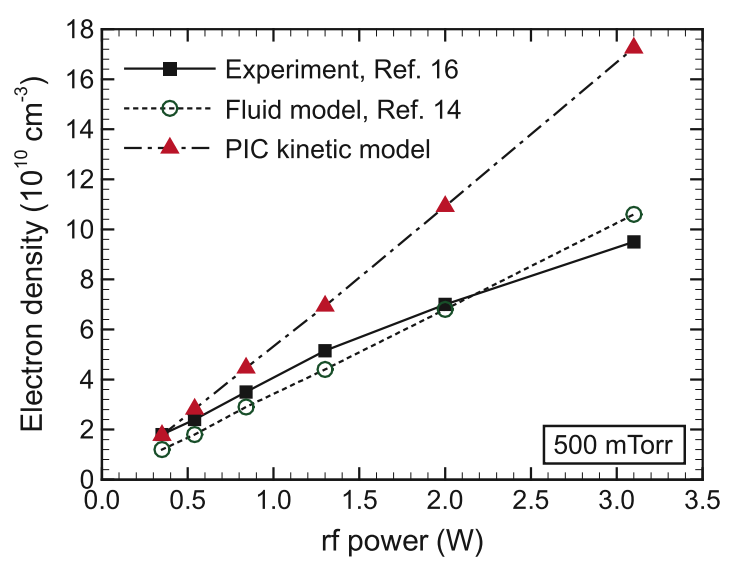

FIG. 9. (Color online) Electron density as a function of rf power at an Ar pressure $p=500$ mTorr and power transfer efficiency $\eta=2.5 \%$ for comparison among the experiment (squares), fluid simulation (circles), and particle simulation based on the kinetic model (deltas).

the experiment. ${ }^{37}$ Given that the dependence of the power transfer efficiency on the rf power was taken into consideration correctly, the electron density obtained at higher rf power would be decreased and closer to the experimental results.

Figure 10 shows the electron density as a function of $\mathrm{Ar}$ pressure for a fixed rf power $P_{\mathrm{rf}}=1.3 \mathrm{~W}$. Here, a power transfer coefficient $\eta=2.5 \%$ is also used for all the pressure conditions, i.e., a specified power deposition of $32.5 \mathrm{~mW}$ is employed. The electron density increases with increasing pressure in the kinetic PIC model, fluid model, and experiment, whereas the electron density decreases with increasing pressure in the collisional PIC model. This discrepancy between the collisional and kinetic model was also demonstrated in conventional low pressure ICP sources for both fluid $^{36}$ and particle simulations. ${ }^{41}$ Figure 11 shows the twodimensional distribution of the electron momentum transfer collision frequency $\nu_{m}$ at a gas pressure of 770 mTorr. Since the angular frequency $\omega$ of $450 \mathrm{MHz}$ is $2.8 \times 10^{9} \mathrm{~s}^{-1}$, the relation of $\nu_{m}<\omega$ is satisfied over the entire plasma region and under all the pressure conditions examined.

In low pressure ICP sources, the collisional heating model is inadequate and the noncollisional heating should be taken into account when the electron collision frequency $\nu_{m}$

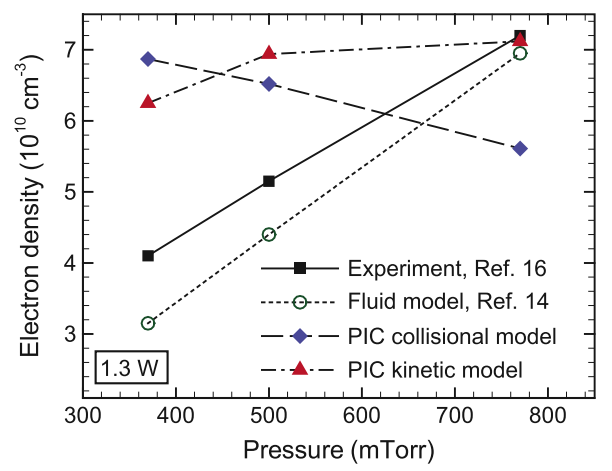

FIG. 10. (Color online) Electron density as a function of Ar pressure at an $\mathrm{rf}$ power $P_{\mathrm{rf}}=1.3 \mathrm{~W}$ and power transfer efficiency $\eta=2.5 \%$ for comparison among the experiment (squares), fluid simulation (circles), particle simulation based on the collisional model (diamonds), and particle simulation based on the kinetic model (deltas). 


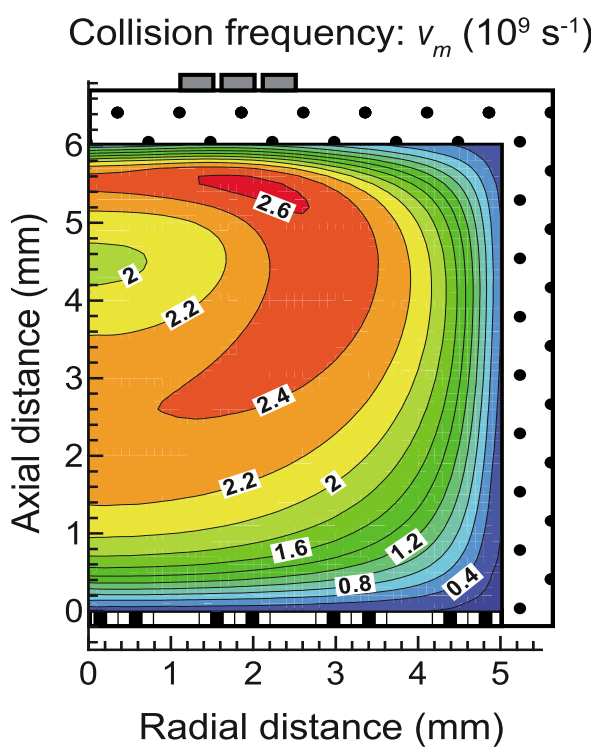

FIG. 11. (Color online) Two-dimensional distribution of the electron momentum transfer collision frequency $\nu_{m}$ in the mICP source based on the kinetic model at an Ar pressure $p=770$ mTorr. Other calculation conditions are the same as those in Fig. 2.

is less than the $\mathrm{rf}$ driving frequency $\omega .^{42,43}$ In such a situation, the plasma density erroneously increases with decreasing pressure if the collisional heating model is employed. ${ }^{36,41}$ This tendency is due to the decrease in coupling efficiency between the rf-electromagnetic fields and the plasma as the pressure decreases. The collisional power deposition is proportional to $\operatorname{Re}\left(\sigma_{p}\right)$, and $\operatorname{Re}\left(\sigma_{p}\right)$ decreases with decreasing pressure because of the lower collision frequency $\nu_{m}$ when $\nu_{m}<\omega$. Larger collisional heating, i.e., higher plasma density is then required to satisfy the constant power deposition. This would be true in our collisional model. It should be noted that the collisional model employed here is essentially identical to the quasicollisional model developed by Rauf and Kushner, ${ }^{36}$ because the collision frequency $\nu_{m}$ in Eq. (9) in the collisional model naturally contains noncollisional effects as shown in Eq. (10). They also reported that noncollisional and fully collisional models had a reasonable agreement in the pressure dependence of the plasma density for Ar but had a poor agreement for other working gases. ${ }^{36}$ The similar trend between the fluid model and experimental data would be fortuitous in Fig. 10.

The noncollisional electron heating is known to be a warm plasma effect. If an electron can traverse the power absorption region (i.e., the skin depth layer) in a time which is shorter than an rf period, then the electron gains net energy from the field. ${ }^{42,43}$ Under our calculation conditions, the rf frequency is excessively high and most of electrons cannot traverse the skin depth layer in an rf period, leading to weak collisionless heating. However, as shown in Fig. 10, the electron density increases with increasing pressure at a constant power deposition in the PIC kinetic model, which implies that collisionless heating would occur to some extent. In such a high rf frequency case, collisionless heating is still dominant at low pressures. ${ }^{44}$ Moreover, the gradient of the electron density in the kinetic model would be larger if the correct power transfer coefficient was employed in the model. In practice the power transfer efficiency is also dependent on pressure, which increases with increasing pressure in the limited pressure range (370-770 mTorr). ${ }^{37}$

\section{CONCLUSIONS}

A two-dimensional particle model for a mICP source in Ar has been developed to investigate the microplasma characteristics and compare the results with available experimental data and fluid simulation results. The mICP source is 5 $\mathrm{mm}$ in inner radius and $6 \mathrm{~mm}$ in length with a planar threeturn coil (5 $\mathrm{mm}$ in diameter). The particle model employed an axisymmetric PIC-MCC and the electromagnetic equation for the rf-induced azimuthal electric field. To couple between the rf-electromagnetic fields and the plasma, two types of power deposition models are used: a collisional model and a kinetic model. The collisional model is based on the coldelectron approximation, where the relation between the plasma current and the electric field is given by Ohm's law, whereas in the kinetic model the current is directly derived by following electron trajectories.

The calculations in the both models were performed for pressures in the range 370-770 mTorr and for rf powers below $3.5 \mathrm{~W}$ at a high of frequency of $450 \mathrm{MHz}$. The results show that the spatial distribution of the plasma density is similar to that in the fluid model, where steep density gradients are obtained. The gradients indicate difficulty in experimental investigations of spatial microplasma distribution. We can find a relatively thick sheath structure compared with the plasma reactor length, where the sheath thickness can reach about $10 \%$ of the plasma reactor length. We also confirm that the electron energy distribution is non-Maxwellian over the entire plasma region. At higher electron energies, the distribution is depleted due to the inelastic collisions. Consequently, the distribution of the electron temperature is quite different from that in the fluid model which neglected the sheath and assumed Maxwellian electrons. Finally, the pressure dependence of the plasma density shows different tendency between the collisional model and the kinetic model. Whereas the electron density increases with increasing pressure in the kinetic model, the electron density decreases with increasing pressure in the collisional model. This discrepancy would be due to noncollisional effects even at high Ar pressures since the electron collision frequency is less than the rf driving frequency.

\section{ACKNOWLEDGMENTS}

We thank Mr. S. Hou and Mr. T. Kato, who used to be members of our laboratory, for refinement of the simulation code for the collisional model. This work was financially supported in part by a Grant-in-Aid for Scientific Research on Innovative Areas Grant No. 21110008 from the Ministry of Education, Culture, Sports, Science, and Technology. One of the authors (Y.T.) was supported by a Grant-in-Aid for Young Scientists Grant No. 21860052 from Japan Society for the Promotion of Science.

\footnotetext{
${ }^{1}$ M. M. Micci and A. D. Ketsdever, Micropropulsion for Small Spacecraft (American Institute of Aeronautics and Astronautics, Reston, 2000).

${ }^{2}$ Y. Takao and K. Ono, Plasma Sources Sci. Technol. 15, 211 (2006).
} 
${ }^{3}$ Y. Takao, K. Ono, K. Takahashi, and K. Eriguchi, Jpn. J. Appl. Phys., Part 145,8235 (2006).

${ }^{4}$ Y. Takao, K. Eriguchi, and K. Ono, J. Appl. Phys. 101, 123307 (2007).

${ }^{5}$ Y. Takao, T. Takahashi, K. Eriguchi, and K. Ono, Pure Appl. Chem. 80, 2013 (2008).

${ }^{6}$ T. Takahashi, Y. Takao, K. Eriguchi, and K. Ono, Phys. Plasmas 16 083505 (2009).

${ }^{7}$ T. Deconinck, S. Mahadevan, and L. L. Raja, J. Appl. Phys. 106, 063305 (2009).

${ }^{8}$ K. H. Becker, K. H. Schoenbach, and J. G. Eden, J. Phys. D 39, R55 (2006).

${ }^{9}$ K. Tachibana, IEEJ Trans. Electr. Electron. Eng. 1, 145 (2006).

${ }^{10}$ R. Foest, M. Schmidt, and K. Becker, Int. J. Mass. Spectrom. 248, 87 (2006).

${ }^{11}$ F. Iza, G. J. Kim, S. M. Lee, J. K. Lee, J. L. Walsh, Y. T. Zhang, and M. G. Kong, Plasma Processes Polym. 5, 322 (2008).

${ }^{12}$ J. Choi, F. Iza, J. K. Lee, and C. M. Ryu, IEEE Trans. Plasma Sci. 35, 1274 (2007).

${ }^{13}$ Y. Ikeda, K. Suzuki, H. Fukumoto, J. P. Verboncoeur, P. J. Christenson, C. K. Birdsall, M. Shibata, and M. Ishigaki, J. Appl. Phys. 88, 6216 (2000).

${ }^{14}$ S. K. Nam and D. J. Economou, J. Appl. Phys. 95, 2272 (2004).

${ }^{15}$ F. Iza, J. K. Lee, and M. G. Kong, Phys. Rev. Lett. 99, 075004 (2007).

${ }^{16}$ J. Hopwood, O. Minayeva, and Y. Yin, J. Vac. Sci. Technol. B 18, 2446 (2000).

${ }^{17}$ H. Takekida and K. Nanbu, IEEE Trans. Plasma Sci. 34, 973 (2006).

${ }^{18}$ C. K. Birdsall, IEEE Trans. Plasma Sci. 19, 65 (1991).

${ }^{19}$ C. K. Birdsall and A. B. Langdon, Plasma Physics via Computer Simulation (Institute of Physics Publishing, Bristol, Philadelphia, 1991).

${ }^{20}$ V. Vahedi and M. Surendra, Comput. Phys. Commun. 87, 179 (1995).

${ }^{21}$ B. W. Yu and S. L. Girshick, J. Appl. Phys. 69, 656 (1991).

${ }^{22}$ X. Chen and E. Pfender, Plasma Chem. Plasma Process. 11, 103 (1991).

${ }^{23}$ H. Fukumoto, I. Fujikake, Y. Takao, K. Eriguchi, and K. Ono, Plasma
Sources Sci. Technol. 18, 045027 (2009).

${ }^{24}$ P. Silvester, Modern Electromagnetic Fields (Prentice-Hall, Englewood Cliffs, 1968).

${ }^{25}$ E. Bultinck, I. Kolev, A. Bogaerts, and D. Depla, J. Appl. Phys. 103, 013309 (2008)

${ }^{26}$ M. Jugroot, Plasma Processes Polym. 6, 360 (2009).

${ }^{27}$ Y. V. Yurgelenas and H. E. Wagner, J. Phys. D 39, 4031 (2006).

${ }^{28}$ J. P. Verboncoeur, J. Comput. Phys. 174, 421 (2001).

${ }^{29}$ Y. J. Hong, M. Yoon, F. Iza, G. C. Kim, and J. K. Lee, J. Phys. D 41, 245208 (2008)

${ }^{30}$ K. Nanbu, IEEE Trans. Plasma Sci. 28, 971 (2000).

${ }^{31}$ M. Surendra, D. B. Graves, and G. M. Jellum, Phys. Rev. A 41, 1112 (1990).

${ }^{32}$ A. Bogaerts, R. Gijbels, and W. Goedheer, Jpn. J. Appl. Phys., Part 1 38, 4404 (1999)

${ }^{33}$ W. H. Cramer, J. Chem. Phys. 30, 641 (1959).

${ }^{34}$ M. A. Lieberman and A. J. Litchenberg, Principles of Plasma Discharges and Materials Processing (Wiley, New York, 1994).

${ }^{35}$ I. V. Schweigert and V. A. Schweigert, Plasma Sources Sci. Technol. 13, 315 (2004).

${ }^{36}$ S. Rauf and M. J. Kushner, J. Appl. Phys. 81, 5966 (1997)

${ }^{37}$ O. B. Minayeva and J. Hopwood, J. Appl. Phys. 94, 2821 (2003).

${ }^{38}$ H. Takekida and K. Nanbu, J. Phys. Soc. Jpn. 73, 756 (2004).

${ }^{39}$ S. K. Nam, D. J. Economou, and V. M. Donnelly, Plasma Sources Sci. Technol. 16, 90 (2007).

${ }^{40}$ J. H. Kim, H. J. Lee, Y. T. Kim, K. W. Whang, and J. H. Joo, J. Vac. Sci. Technol. A 15, 564 (1997).

${ }^{41}$ H. Takekida and K. Nanbu, Thin Solid Films 506-507, 729 (2006).

${ }^{42}$ M. M. Turner, Phys. Rev. Lett. 71, 1844 (1993).

${ }^{43}$ M. M. Turner, J. Phys. D 42, 194008 (2009).

${ }^{44}$ V. Vahedi, M. A. Lieberman, G. DiPeso, T. D. Rognlien, and D. Hewett, J. Appl. Phys. 78, 1446 (1995) 